\title{
An Efficient Classification Algorithms for Image Retrieval Based Color and Texture Features
}

\author{
Iman Abduljabbar Saad \\ Electronic Computer Center \\ Al-Mustansiriyah University, Baghdad, Iraq
}

Recived : 2611 $\mid 2017$

Revised : 11\12\2017

Accepted : 14\12\2017

Available online : $26 / 1 / 2018$

DOI: $10.29304 / j q c m .2018 .10 .1 .350$

\begin{abstract}
:
Content-Based Image Retrieval CBIR system commonly extracts retrieval results respecting to the similarities of the extracted feature of the given image and the candidate images. The proposed system presented a comparative analysis of five types of classifiers which used in CBIR. These classifiers are Multilayer Perceptron (MP), Sequential Minimal Optimization (SMO), Random Forest (RF), Bayes Network (BN) and Iterative Classifier Optimizer (ICO). It has been investigated to find out the best classifier in term of performance and computation to be the suitable for image retrieval. The low level image features which include texture and color are used in the proposed system. The color features involve color-histogram, color-moments and color-autocorrelogram while texture features involve wavelet transform and log Gabor filter. Also the system will include hybrid of texture and color features to get efficient image retrieval. The system was tested using WANG database, and the best average precision achieved was $(85.08 \%)$ when combining texture and color features and using the (RF) classifier.
\end{abstract}

Keywords: - Color descriptors, Textures descriptors, Classifiers algorithms.

\section{Introduction}

Nowadays, there have been major developments in image acquisition techniques as well as the expansion of data storage units for very huge databases. In order to retrieve and manage this large number of images with high efficiency, an effective retrieval system was necessary [1]. The method of automatic image annotation is used for automatically generating number of labels for describing the image content. Low level features typically used for images annotated with labels. Machine learning techniques provided facilitates to annotate the image by learning the correlation between features of image and annotated labels [2].
A large number of researchers aim to retrieve images based on its content and tried to automate the process of image analysis. Problems related to the image retrieval consist of two sections; (feature extraction and classification). Many different techniques and algorithms have been used by lot of researchers to extract image features and classification to improve recall and accuracy [3].

Image retrieval method, which depends primarily on the image content, has been suggested in this paper to find related images. The visible image's content is analyzed based on low level features that obtained from the image. Based on methods of automatic image annotation, there are various algorithms for automated image retrieval and classifications are used. 


\section{Related Work}

This section of the paper includes some published researches written in the field of CBIR.

[4] Proposed method depend on the color moments features and Wang database for an image retrieval. In this method the image is partitioned into four parts. After that, the color moments are extracted of each part and clustered into four classes. The mean moment is extracted for each class and considered as such as an image primitive. The entire primitives are applied as features and every class mean is combined into a one class mean. In this work the average precision gain was 31.09

[5] Proposed method used texture and color for CBIR technique. They used Zernike chromaticity distribution to capture color features. Where, texture features are extracted using a rotationinvariant and scale-invariant image descriptor in Contourlet domain. The average precision gain in this work was 51.02.

[6] Proposed a new method depend on 2D DualTree Discrete Wavelet Transform (2D DT DWT). In the first, all images are converted from RGB color space to $\mathrm{YCbCr}$ color space. Then, the 2D DT DWT is applied to each color band. For all images in the database, the features such as mean, energy, entropy and standard deviation are extracted from each color bands of the image. The Euclidean distance is used in the image matching process to find the closer relevant images. WANG database was used and the average precision gain in this work was 33.86 .

[7] Proposed method used neural network based back propagation and global image properties. At first, the neural network is trained about the images' database features. Image's features that considered in this method are GLCM (Gray Level Cooccurrence Matrix), color histogram and edge histogram. The results are shown an improvement in image retrieval about precision and recall.

[8] Proposed method depended on color and texture features and used Discrete Wavelet Transform and the Self Organizing Map (SOM) artificial neural networks. The average precision when using WANG database was 55.88 .
[9] In this paper, the performance of Sparse Banded Filter matrices are evaluated by comparing it with the standard edge detection filters through CBIR. 7 classes out of the 10 classes of WANG database are used. The features are extracted depended on edge detection and Singular Value Decomposition. The average precision when using WANG database was $23.69 \%$.

[10] Proposed approach for CBIR based on color and texture data. In this regard, color histogram and color moment are used as color feature; further, PCA statistical method is applied to reduce dimensions. The average precision when using WANG database was $62.4 \%$.

This paper is organized as follows: The first section of the paper presents the proposed method. The second section discusses the experimental result .Finally, in the last section; the main conclusion concerning the proposed approach is given.

\section{Proposed Method}

In this work, two types of features and five types of classifier are used to compare their performances in increase image retrieval accuracy. The first type of feature is color features which include HSV histogram, color moments and color Autocorrelogram, while second type is texture features which based on transform method that includes Gabor wavelet and Wavelet transforms. The classification algorithms that used in this work are Iterative Classifier Optimizer (ICO), Bayes Network (BN), Random Forest (RF), Multilayer Perceptron (MP) and Sequential Minimal Optimization (SMO). Figure (1) shows the diagram of proposed method. 


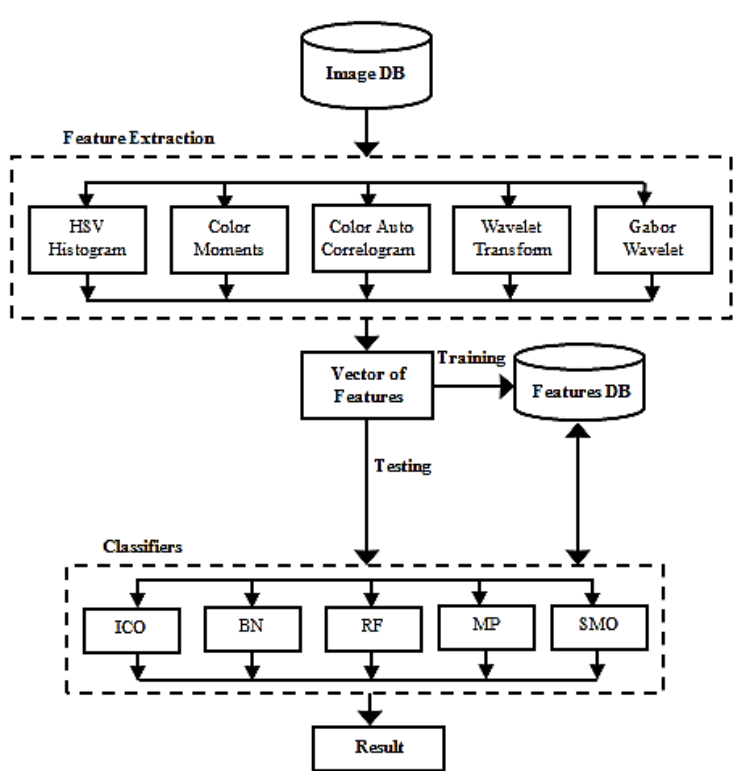

Figure (1): Block Diagram of Proposed Method.

\section{Color Feature Extraction}

For extraction of color feature, three different methods have been used; histogram, autocorrelogram and color moments which describe as follows:

\section{A. HSV color space}

HSV space is a way to describe color that considered more intuitive than other color spaces. HSV contains three components (Hue, Saturation and Value) [11]. HSV color space is derived from RGB values using the following equations [12]:

$$
\begin{aligned}
& H=\cos ^{-1}\left\{\frac{\frac{1}{2}[(R-G)+(R-B)]}{\sqrt{(R-G)^{2}+(R-B)(G-B)}}\right\} \\
& S=1-\frac{3}{R+G+B}\lfloor M I N(R, G, B)\rfloor \\
& V=\frac{1}{3}(R+G+B)
\end{aligned}
$$

The proposed method computes histogram for each band separately such as $H$ band, $S$ band and $V$ band. The histogram count number of pixels corresponds to a specific color in quantized color's space. To reduce computing time required for histogram, the color space must be quantizing into a limited number of levels. HSV color space quantized by using uniform quantization from 360 levels into 8 levels for $H$ while 2 levels for each $S$ and $V$.

\section{B. Color Moments}

The ground of the color moments depends on the color distribution in the image which represents probability distribution that can be distinguished by different moments. Color moments can be classified into number of order as follows: first order, second order, third order and higher orders. The mean (first order) and standard deviation (second order) color moments have been used in the proposed method. They are extracted for each band of RGB color space; Red, Green and Blue bands [2]. Mathematically, the early two moments are described as follow:

$u_{i}=\frac{1}{N} \sum_{j=1}^{N} f_{i j}$

$\sigma_{i}=\frac{1}{N} \sum_{j=1}^{N}\left(\left(f_{i j}-u_{i}\right)^{2}\right)^{\frac{1}{2}}$

Where $N$ represents the number of pixels in image, $f_{i j}$ represents the value of $i^{\text {th }}$ color component of the image pixel $j$ [13].

\section{Color Autocorrelogram}

Color autocorrelogram, is counseled to describe not merely the color allocations of pixels, but additionally the spatial correlation of color's pairs. The elements in the autocorrelograms are frequencies of the occurrence of the two identical color levels at a given distance. In proposed approach autocorrrelogram is used because of its smaller size [13]. For faster retrieval, the color is quantized to 64 colors.

\section{Texture Feature Extraction}

For extraction texture feature, two different methods have been used; discrete wavelet transform and $\log$ Gabor filter.

\section{A. Discrete Wavelet Transform}

The wavelet transforms commutated for a $2 \mathrm{D}$ signal level by using sub-sampling and recursive filtering. The signal for each level decomposed into four frequency sub bands, Low-Low, Low-High, High-Low and High-High [13]. 
This signal is decomposed into two ranges when passing through these filters: the low-pass filter and high-pass filter. Rough information of the signal is extracted by the low-pass filter that corresponds to the average operation while detailed information is extracted from the signal using a high-pass filter that corresponds to the variation operation [14]. In the proposed method four level of decomposition is applied.

\section{B. Gabor Wavelet}

The Gabor Wavelet consists of a group of Gabor filters which a set of wavelets capturing energy at different frequencies and directions. Localized frequency description is provided from signal expansion. Texture features is extracted from capturing local features-energy from this signal. So, Gabor wavelet has good characteristics in space position, space frequency and direction selectivity [13].

\section{Classifier Types}

In this work, there are five types of classification algorithms are used and it is described as follow:

\section{A. Sequential Minimal Optimization (SMO)}

Sequential Minimal Optimization algorithm is used for training a support vector classifier. It is a simple algorithm that used for quickly solves the Support Vector Machine Quadratic Programming (SVM QP) problem. The SMO does not require any extra matrix storage and no numerical QP optimization. At every step, SMO chooses two Lagrange multipliers to jointly optimize, finds the optimal values for these multipliers, and updates the SVM to reflect the new optimal values. The advantage of SMO lies in the fact that solving for two Lagrange multipliers can be done analytically [15].

\section{B. Random Forest $(\mathrm{RF})$}

Random Forest is a set learning methods that used for regression and classification. The basic concept of the random forest's methodology is the constructing a forest of random decision trees; which are created by randomizing the spilt at each node of decision tree. Each tree is likely to be less accurate than a tree created with exact splits. But, by combining several of these "approximate" trees in an ensemble, we can improve the accuracy, often doing better than a single tree with exact split [16] [17].

\section{Bayes Network $(B N)$}

Bayes Network is called Naïve Bayes algorithm which is based on Bayes theorem; it is useful for large data sets. Attributes are independent, which is presence of one particular feature in a class, is independent of any other features in that class. This is a statistical based learning algorithm; it is efficient, strong, fast and considerably accurate in real world scenario [18], this algorithm works better on categorical data, easily handle missing data. Dimensionality of data is very high and helps in solving predictive problems; there is no repetitive parameter estimation [19].

\section{Iterative Classifier Optimizer (ICO)}

Iterative classifier uses cross validation and optimizes the number of iteration for the given classifier; it is capable of handling missing, nominal, binary classes and attributes like numeric, nominal, binary, empty nominal [20]. In this paper test, ICO is used 10 iterations.

\section{E. Multilayer Perceptron (MP)}

Multilayer Perceptron is classifier based on using back propagation for classification. MP is a feed-forward neural network composed of an input layer of nodes, two or more layers of perceptron and the output layer. The layers between the input and output layers are called hidden layers [21]. It depends on supervised learning process which conducted through change the connection weights after handling each data's piece, depending on the amount of error that results in the output target and compared with the expected results. It has a lot of successful applications in solving complex problems in the real world [22]. 


\section{Feature selection}

The most important issues in the field of retrieving images is found features vector that have a great power of distinction. In practice there are a larger numbers of candidate features are extracted, then the best of them is selected. In this paper, to select the best set of features, the CFS (Correlation based Feature Selection) algorithm is used. The selected features set has low inter correlation and very correlated with the class [23].

\section{Experimental Result}

This section was devoted to present and discuss several classification algorithms to compare their performance in image retrieval accuracy. These classification algorithms are Multilayer Perceptron (MP), Sequential Minimal Optimization (SMO),
Random Forest (RF), Bayes Network (BN) and Iterative Classifier Optimizer (ICO). The process of classification is performed by three algorithms based on color features, texture features and combination of them. These three algorithms produced different results which are explained in this section.

The WANG database is used which consist of 1000 images belong to 10 different classes where each class includes 100 images of 384 x 256 pixels dimension, Figure (2) shows examples of WANG database. The database is divided into two categories: Train Data and Test Data; test dataset contain the images which we want to Query (in this work $20 \%$ from each class images are used for testing) and train dataset contain the images that the user wants (in this work $80 \%$ from each class images are used for training).

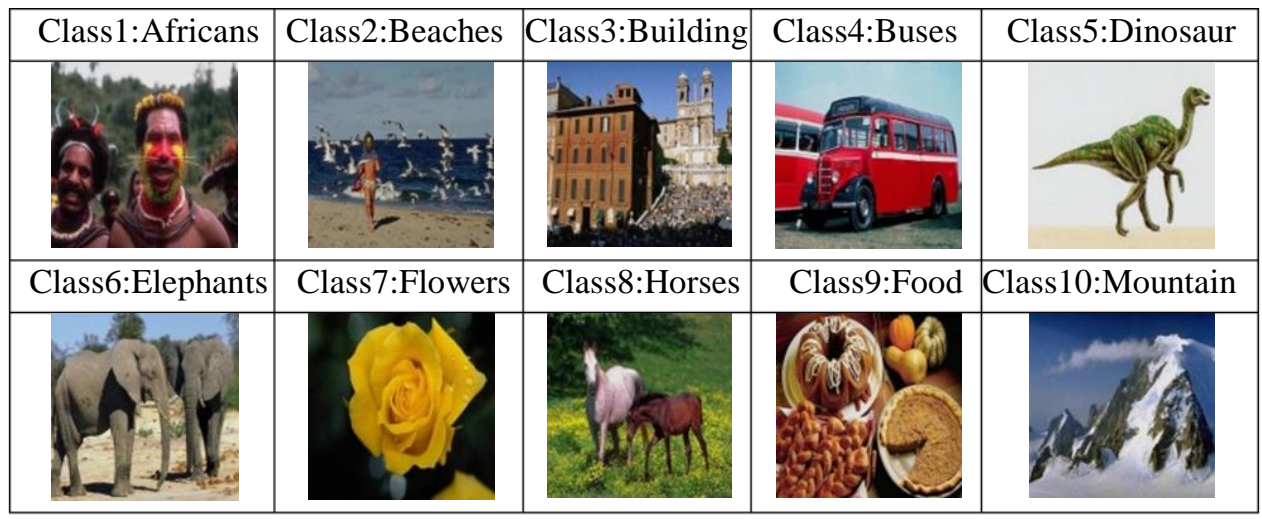

Figure (2): Example Image of WANG Database classes.

Retrieval results are examined by the known "precision and recall" criteria, which are described as follows:

Precision $=$ no. correct of image $/$ no. retrieval images

Recall $=$ no. correct of image/no. all image related 


\section{A. Implemented Color Features}

In the first proposed algorithm, the training and testing have been done on features extracted from various color components; HSV Histogram, Autocorrelogram and Color Moments. With HSV Histogram, for saving the time, each of $H, S$ and $V$ is quantized to be equivalently to $8 \times 2 \times 2$ and get a 32-dimensional color feature vector.
Where with Color Autocorrelogram, the image is quantized into 64 colors $=4 \times 4 \times 4$ in $\mathrm{RGB}$ space and four predefined distances between neighbour pixel intensities [ $\left[\begin{array}{llll}1 & 3 & 5 & 7\end{array}\right]$, so, there is 64dimensional color feature vector. In case of using Color Moments, first two color moments are extracted from each one of (Red, Green and Blue) planes of image that will led to 6-dimensional color feature vector. The results of the Precision and Recall are shown in Table (1) depending on the color features vector which consists of 102 features.

Table (1): Precision and Recall results for Classification Techniques Using Color Features.

\begin{tabular}{|c|c|c|c|c|c|c|c|c|c|c|}
\hline \multirow{3}{*}{ Classes } & \multicolumn{10}{|c|}{ Classifier Type } \\
\hline & \multicolumn{2}{|c|}{ MP } & \multicolumn{2}{|c|}{ SMO } & \multicolumn{2}{|c|}{ RF } & \multicolumn{2}{|c|}{ BN } & \multicolumn{2}{|c|}{ ICO } \\
\hline & Precision $\%$ & Recall\% & Precision $\%$ & Recall\% & Precision $\%$ & Recall\% & Precision $\%$ & Recall\% & Precision $\%$ & Recall\% \\
\hline Africans & 70.6 & 70.6 & 56.5 & 76.5 & 70 & 82.4 & 77.8 & 82.4 & 70 & 82.4 \\
\hline Beaches & 73.7 & 60.9 & 80 & 69.6 & 76 & 82.6 & 73.9 & 73.9 & 63.3 & 82.6 \\
\hline Buildings & 72.2 & 68.4 & 65 & 68.4 & 60.9 & 73.7 & 73.7 & 73.7 & 56.5 & 68.4 \\
\hline Buses & 55.6 & 66.7 & 66.7 & 66.7 & 91.7 & 73.3 & 100 & 73.3 & 69.2 & 60 \\
\hline Dinosaurs & 100 & 100 & 100 & 100 & 93.8 & 100 & 93.8 & 100 & 100 & 100 \\
\hline Elephants & 73.1 & 82.6 & 85 & 73.9 & 85 & 73.9 & 84 & 91.3 & 76.5 & 56.5 \\
\hline Flowers & 100 & 84.6 & 95.8 & 88.5 & 100 & 88.5 & 95.8 & 88.5 & 92.6 & 96.2 \\
\hline Horses & 85.7 & 100 & 73.9 & 94.4 & 85.7 & 100 & 90 & 100 & 82 & 100 \\
\hline Foods & 71.4 & 62.5 & 63.2 & 50 & 72.7 & 66.7 & 70.8 & 70.8 & 68.8 & 45.8 \\
\hline Mountains & 69.6 & 80 & 76.2 & 80 & 88.9 & 80 & 85 & 85 & 82.4 & 70 \\
\hline $\begin{array}{l}\text { Average } \\
\text { Precision }\end{array}$ & 77.19 & 77.63 & 76.23 & 76.80 & 82.47 & 82.11 & 84.48 & 83.89 & 76.11 & 76.19 \\
\hline $\begin{array}{r}\text { Model Build } \\
\text { Time(Second) }\end{array}$ & \multicolumn{2}{|c|}{33.3} & \multicolumn{2}{|c|}{0.46} & \multicolumn{2}{|c|}{0.77} & \multicolumn{2}{|c|}{0.18} & \multicolumn{2}{|c|}{9.99} \\
\hline
\end{tabular}

According to the output results listed in Table (1), when applying algorithm on the tested dataset, indicate that the best average (precision $=84.48 \%$, recall $=83.89 \%$ ) has been gain when using $\mathrm{BN}$ classifier with low time $(0.18)$ second. It is noted that class4 (Buses) of images have higher precision rate (100) with just BN classifiers type, class5 (Dinosaurs) have higher precision and recall rate (100\%) for most classifiers types because these images have simple background, therefor the nature of images has good effect on the results. Class7 (Flowers) have higher precision rate $(100 \%)$ for MP and RF classifiers types, where class8 (Horses) of images have higher recall rate for all classifiers types except SMO.

\section{B. Implemented Texture Features}

In the second algorithm, the training and testing have been done on texture features. Gabor filters are applied on the image with four scales of frequency and six orientations. The Mean-squared energy and mean amplitude for each scale and orientation is calculated and created a texture feature vector of length 48. When applying Wavelet, fourth-level decomposition is achieved, so, $1 \times 20$ feature vector containing the first two moments (mean and standard deviation) of wavelet's coefficients are created and consist of 40 features. The results of the Precision and Recall are show in Table (2) depending on the texture features vector which consists of 88 features, when applying algorithm on the tested images. 
Table (2): Precision and Recall results for Classification Techniques Using Textures Features.

\begin{tabular}{|c|c|c|c|c|c|c|c|c|c|c|}
\hline \multirow{3}{*}{ Classes } & \multicolumn{10}{|c|}{ Classifier Type } \\
\hline & \multicolumn{2}{|c|}{ MP } & \multicolumn{2}{|c|}{ SMO } & \multicolumn{2}{|c|}{ RF } & \multicolumn{2}{|c|}{ BN } & \multicolumn{2}{|c|}{ ICO } \\
\hline & Precision $\%$ & Recall\% & Precision $\%$ & Recall\% & Precision $\%$ & Recall\% & Precision $\%$ & Recall\% & Precision\% & Recall\% \\
\hline Africans & 66.7 & 47.1 & 52.9 & 52.9 & 55.6 & 58.8 & 35 & 41.2 & 31.6 & 35.3 \\
\hline Beaches & 73.1 & 82.6 & 54.2 & 56.5 & 56 & 60.9 & 53.6 & 65.2 & 63.2 & 52.2 \\
\hline Buildings & 66.7 & 63.2 & 55.6 & 52.6 & 63.2 & 63.2 & 50 & 36.8 & 50 & 57.9 \\
\hline Buses & 73.7 & 93.3 & 78.6 & 73.3 & 86.7 & 86.7 & 54.2 & 86.7 & 72.7 & 53.3 \\
\hline Dinosaurs & 100 & 100 & 100 & 100 & 100 & 100 & 100 & 100 & 100 & 100 \\
\hline Elephants & 83.3 & 87 & 70.4 & 82.6 & 71.4 & 87 & 68.2 & 65.2 & 69.6 & 69.6 \\
\hline Flowers & 89.3 & 96.2 & 89.3 & 96.2 & 96 & 92.3 & 92.3 & 92.3 & 100 & 96.2 \\
\hline Horses & 88.9 & 88.9 & 73.7 & 77.8 & 76.5 & 72.2 & 87.5 & 77.8 & 83.3 & 83.3 \\
\hline Foods & 82.4 & 58.3 & 56.3 & 37.5 & 50 & 29.2 & 43.8 & 29.2 & 47.6 & 41.7 \\
\hline Mountains & 56.5 & 65 & 59.1 & 65 & 58.3 & 70 & 42.1 & 40 & 44.4 & 60 \\
\hline $\begin{array}{l}\text { Average } \\
\text { Precision } \\
\end{array}$ & 78.06 & 78.16 & 69.01 & 69.44 & 71.37 & 72.03 & 62.67 & 63.44 & 66.24 & 64.95 \\
\hline $\begin{array}{r}\text { Model Build } \\
\text { Time(Second) }\end{array}$ & \multicolumn{2}{|c|}{23.1} & \multicolumn{2}{|c|}{0.21} & \multicolumn{2}{|c|}{0.63} & \multicolumn{2}{|c|}{0.07} & \multicolumn{2}{|c|}{9.32} \\
\hline
\end{tabular}

The results listed in Table (2), show that the high average (precision $=78.06 \%$, recall $=78.16 \%$ ) has been gain when using MP classifier with time (23.1) second. Also, it is noted that class5 (Dinosaurs) of images have higher precision and recall rate $(100 \%)$ for all classifiers types because these images have simple background, where, class7 (Flowers) of images have higher precision rate for ICO classifiers type. The all classifier types which based on color features gave better results on all classes than classifier types which based on texture features.

\section{Implemented Hybrid Features}

In the third algorithm, for enhancing system's performance of the image retrieval, training and testing have been done with combination of color and textural features (Hybrid). The results of the Precision and Recall are show in Table (3) depending on the features vector of color and texture which consists of 190 features.

Table (3): Precision and Recall results for Classification Techniques Using Hybrid Features.

\begin{tabular}{|c|c|c|c|c|c|c|c|c|c|c|}
\hline \multirow{3}{*}{ Classes } & \multicolumn{10}{|c|}{ Classifier Type } \\
\hline & \multicolumn{2}{|c|}{ MP } & \multicolumn{2}{|c|}{ SMO } & \multicolumn{2}{|c|}{ RF } & \multicolumn{2}{|c|}{$\mathbf{B N}$} & \multicolumn{2}{|c|}{ ICO } \\
\hline & Precision \% & Recall\% & Precision $\%$ & Recall\% & Precision $\%$ & Recall\% & Precision\% & Recall\% & Precision $\%$ & Recall\% \\
\hline Africans & 82.4 & 82.4 & 54.2 & 76.5 & 76.9 & 58.8 & 75 & 70.6 & 80 & 70.6 \\
\hline Beaches & 69.2 & 78.3 & 80 & 87 & 67.7 & 91.3 & 65.4 & 73.9 & 75. & 78.3 \\
\hline Buildings & 81.3 & 69.4 & 85.7 & 63.2 & 70 & 73.7 & 47.6 & 52.6 & 61.5 & 84.2 \\
\hline Buses & 93.3 & 93.3 & 92.3 & 80 & 83.3 & 100 & 76.5 & 86.7 & 78.6 & 73.3 \\
\hline Dinosaurs & 100 & 100 & 100 & 100 & 100 & 100 & 100 & 100 & 100 & 100 \\
\hline Elephants & 90.9 & 87 & 87 & 87 & 90.9 & 87 & 80 & 87 & 81.8 & 78.3 \\
\hline Flowers & 100 & 100 & 96.3 & 100 & 100 & 96.2 & 100 & 96.2 & 96.2 & 96.2 \\
\hline Horses & 96.7 & 100 & 94.7 & 100 & 94.7 & 100 & 93.8 & 83.3 & 85.7 & 100 \\
\hline Foods & 76.2 & 74.7 & 85 & 70.8 & 85.7 & 50 & 55.6 & 41.7 & 70.6 & 50 \\
\hline Mountains & 78.9 & 85 & 85 & 85 & 78.3 & 90 & 81 & 85 & 95 & 95 \\
\hline $\begin{array}{l}\text { Average } \\
\text { Precision }\end{array}$ & 86.9 & 87 & 86 & 85 & 84.8 & 84.7 & $77.5 \%$ & 77.7 & 82.4 & 82.6 \\
\hline $\begin{array}{c}\text { Model Build } \\
\text { Time(Second) }\end{array}$ & \multicolumn{2}{|c|}{58.2} & \multicolumn{2}{|c|}{0.55} & \multicolumn{2}{|c|}{1.77} & \multicolumn{2}{|c|}{0.2} & \multicolumn{2}{|c|}{18.9} \\
\hline
\end{tabular}


The results listed in Table (3), show that the high weighted average (precision $=86.9 \%$, recall $=87 \%$ ) has been gain when using MP classifier with time (58.2) second. Also, it is noted that class4 (Buses) of images have higher recall rate (100) with just RF classifiers type, class5 (Dinosaurs) of images have higher precision and recall rate $(100 \%)$ for all classifiers types, class7 (Flowers) of images have higher precision and recall rate $(100 \%)$ for all classifiers types except ICO, where Class8 (Horses) of images have higher recall rate $(100 \%)$ for all classifiers types except $\mathrm{BN}$.

Table (4): Precision and Recall results for Classification Techniques Using Part of Hybrid Features.

\begin{tabular}{|c|c|c|c|c|c|c|c|c|c|c|}
\hline \multirow{3}{*}{ Classes } & \multicolumn{10}{|c|}{ Classifier Type } \\
\hline & \multicolumn{2}{|c|}{ MP } & \multicolumn{2}{|c|}{ SMO } & \multicolumn{2}{|c|}{ RF } & \multicolumn{2}{|c|}{$\mathbf{B N}$} & \multicolumn{2}{|c|}{ ICO } \\
\hline & Precision\% & Recall\% & Precision $\%$ & Recall\% & Precision $\%$ & Recall\% & Precision $\%$ & Recall\% & Precision $\%$ & Recall\% \\
\hline Africans & 78.6 & 64.7 & 72.2 & 76.5 & 82.4 & 82.4 & 82.4 & 82.4 & 73.3 & 64.7 \\
\hline Beaches & 85.7 & 78.3 & 72 & 78.3 & 70.8 & 73.9 & 63 & 73.9 & 70.8 & 73.9 \\
\hline Buildings & 72.2 & 68.4 & 81.3 & 68.4 & 73.7 & 73.7 & 61.1 & 57.9 & 60 & 78.9 \\
\hline Buses & 73.7 & 93.3 & 92.9 & 86.7 & 86.7 & 86.7 & 93.3 & 93.3 & 84.6 & 73.3 \\
\hline Dinosaurs & 100 & 100 & 100 & 100 & 100 & 100 & 100 & 100 & 100 & 100 \\
\hline Elephants & 80.8 & 91.3 & 76.9 & 87 & 87.5 & 91.3 & 72.4 & 91.3 & 80.8 & 91.3 \\
\hline Flowers & 96 & 92.3 & 96.3 & 100 & 100 & 96.2 & 96.3 & 100 & 100 & 100 \\
\hline Horses & 94.4 & 94.4 & 90 & 100 & 88.2 & 88.9 & 94.1 & 88.9 & 94.7 & 100 \\
\hline Foods & 71.4 & 62.5 & 78.9 & 62.5 & 78.9 & 62.5 & 73.3 & 45.8 & 75 & 50 \\
\hline Mountains & 69.6 & 80 & 85 & 85 & 82.6 & 95 & 90 & 90 & 85.7 & 90 \\
\hline $\begin{array}{l}\text { Average } \\
\text { Precision }\end{array}$ & 82.2 & 82.52 & 84.6 & 84.44 & 85.08 & 85.06 & 82.6 & 82.35 & 82.5 & 82.21 \\
\hline $\begin{array}{c}\text { Model Build } \\
\text { Time(Second) }\end{array}$ & \multicolumn{2}{|c|}{15.55} & \multicolumn{2}{|c|}{0.42} & \multicolumn{2}{|c|}{0.48} & \multicolumn{2}{|c|}{0.07} & \multicolumn{2}{|c|}{6.19} \\
\hline
\end{tabular}

The results listed in Table (4), show that the high average (precision $=85.08 \%$, recall $=85.06 \%$ ) has been gain when using RF classifier with time (0.48) second. Also, it is noted that class5 (Dinosaurs) of images have higher precision and recall rate $(100 \%)$ for all classifiers types, where class7 (Flowers) of images have higher precision and recall rate for most classifiers types, Class8 (Horses) of images have higher recall rate $(100 \%)$ for two classifiers types SMO and ICO.
In order to reduce the computation time, the selection feature algorithm is used to find best feature vector. The length of feature vector is reduced from 190 features to 59 best features. The results of the Precision and Recall are show in Table (4) depending on the features vector of the best color and texture features, when applying algorithm on tested images.
General averages of precision and recall when using each classifier type (MP, SMO, RF, BN and ICO) with each feature type (color features, texture features and hybrid) for all 10 classes of the dataset is plotted, as shown in Figures (3) and (4). 


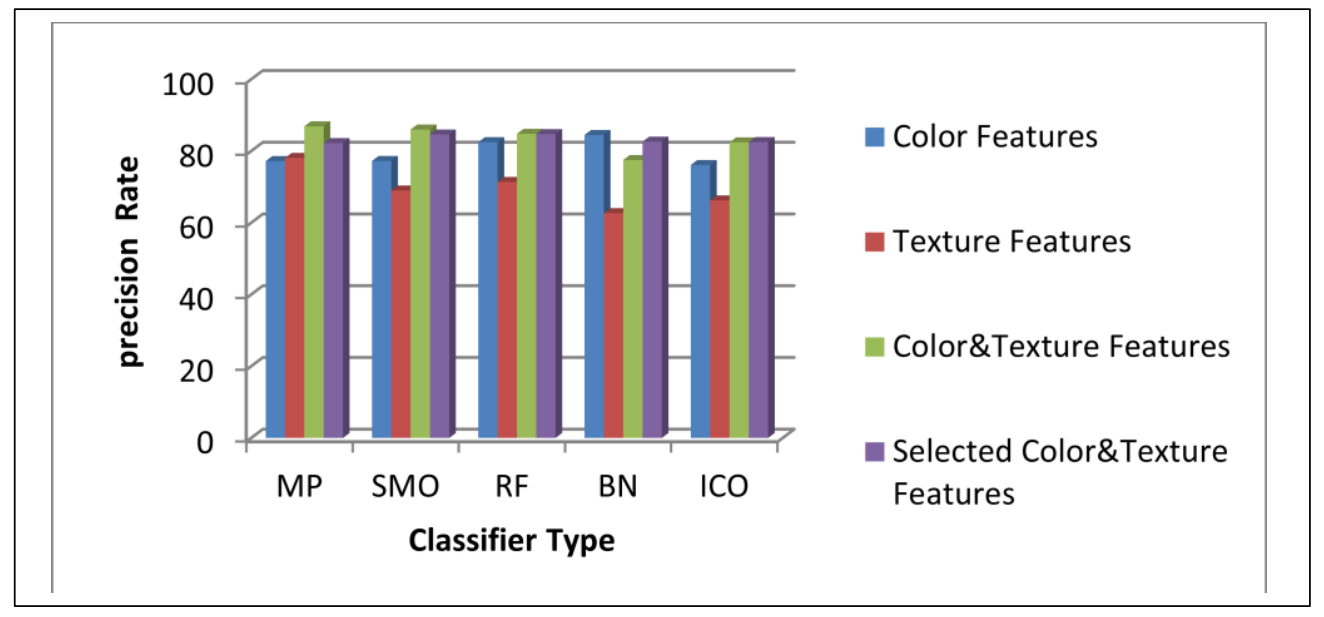

Figure (3): Bar graph showing the average of precision per classifier type.

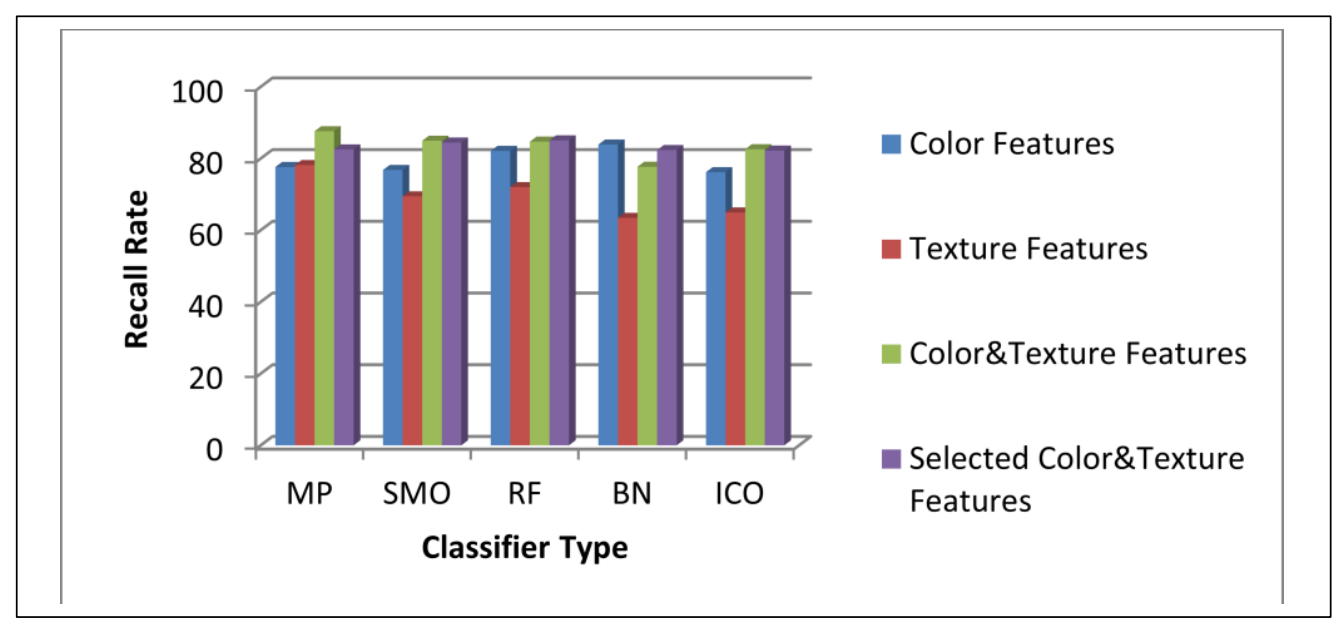

Figure (4): Bar graph showing the average of Recall per classifier type.

Figures (3) and (4) show that, high average (precision $\&$ recall) results of this system were when hybrid of color and textures features along with MP classifier is used, and when using selected best features of color and textures along with RF classifier type.

\section{Conclusions}

In this work, the results show that all classifiers types based on hybrid features of color and texture have given better results than using color features only or texture features only in image retrieval because color information and relative position of pixels are considered very important. The results of the average of precision and recall showed that
MP classifier type was the best one from the others in the image retrieval with high complexity time when using all color and textures features. Where, the RF classifier type was the best one from the others in the average of precision and recall with low complexity time, when using the best selected features of the color and textures. Also, it's found that the complexity of the image is very affected on the image retrieval results. Table (5) lists the average precision values attained by the proposed method compared with those given in previous studies, taking into consideration that in these studies same datasets have been used. The listed results demonstrate that the proposed method in this paper outperforms other methods. 
Table (5): A Comparison between the performance results of proposed method with other methods applied on the same database.

\begin{tabular}{|c|c|c|c|c|c|c|}
\hline Classes & $\begin{array}{c}\text { Precision\% } \\
{[\mathbf{4}]}\end{array}$ & $\begin{array}{c}\text { Precision\% } \\
{[\mathbf{5}]}\end{array}$ & $\begin{array}{c}\text { Precision\% } \\
{[\mathbf{6}]}\end{array}$ & $\begin{array}{c}\text { Precision\% } \\
{[\mathbf{8}]}\end{array}$ & $\begin{array}{c}\text { Precision\% } \\
{[\mathbf{1 0}]}\end{array}$ & $\begin{array}{c}\text { Precision\% } \\
\text { Proposed } \\
\text { Method }\end{array}$ \\
\hline Africans & 13.3 & 40.9 & 27 & 27.8 & 55 & 82.4 \\
\hline Beaches & 26.15 & 39.4 & 33.4 & 54.2 & 48 & 70.8 \\
\hline Buildings & 11.05 & 30.5 & 35 & 34.4 & 38 & 73.7 \\
\hline Buses & 17.25 & 73.7 & 32.2 & 52.6 & 60 & 86.7 \\
\hline Dinosaurs & 17.25 & 58.8 & 30.4 & 52.6 & 93 & 100 \\
\hline Elephants & 34.9 & 49.1 & 38.4 & 55.6 & 52 & 87.5 \\
\hline Flowers & 49.5 & 71.1 & 29.6 & 82.8 & 77 & 100 \\
\hline Horses & 20.8 & 52.5 & 34.6 & 74.8 & 83 & 88.2 \\
\hline Foods & 15.6 & 31.2 & 40 & 30.4 & 70 & 78.9 \\
\hline Mountains & 25.9 & 63 & 38 & 50 & 48 & 82.6 \\
\hline $\begin{array}{c}\text { Average } \\
\text { Precision }\end{array}$ & $\mathbf{2 3 . 1 7}$ & $\mathbf{5 1 . 0 2}$ & $\mathbf{3 3 . 8 6}$ & $\mathbf{5 1 . 5 2}$ & $\mathbf{6 2 . 4}$ & $\mathbf{8 5 . 0 8}$ \\
\hline
\end{tabular}

\section{References}

[1] M. Waghmare and K Patidar, "A System for the Retrieval of Images from Large Database", International Journal on Recent and Innovation Trends in Computing and Communication, Vol. 4, Issue.1, PP. 26 - 29, 2016.

[2] N. S. Naik, S. S. Kulkarni and A. L. Kadam, "Content Based Image Retrieval Using Color, Texture and Hybrid Features", International Journal of Engineering Research and Development, Vol. 12, Issue 6, PP.31-38, June 2016.

[3] S. A. Husain and F. S. Akbar, "A Comparative Analysis of Feature Sets for Image Classification Using Back Propagation Neural Network", International Journal of Information and Electronics Engineering, Vol. 5, No. 1, January 2015.

[4] H. H. Bhuravarjula and V.N.S. Kumar, "A Novel Content Based Image Retrieval Using Variance Color Moment", International Journal of Computational Engineering Research, Vol.1, PP.93-99, 2012.

[5] X. Wang, H.Yang and D.Li ," A new contentbased image retrieval technique using color and texture information", Computers \& Electrical Engineering, Vol.39, No.3, PP.746-761, 2013.
[6] S.K. Thirunavuk, R.P. Ahila, S. Arivazhagan and C. Mahalakshmi, "Content Based Image Retrieval Based on Dual Tree Discrete Wavelet Transform", International Journal of Research in Computer and Communication Technology, Vol.2, PP.473-477, 2013.

[7] A. Nagathan and I. Manimozhi, "ContentBased Image Retrieval System using FeedForward Backpropagation Neural Network", International Journal of Computer Science Engineering, Vol. 2 No.04, July 2013.

[8] A. Huneiti and M. Daoud, "Content-Based Image Retrieval Using SOM and DWT", Journal of Software Engineering and Applications, Vol.8, PP.51-61, 2015.

[9] H .T. Suseelan, S. Sudhakaran, V. Sowmya and Dr. K. P. Soman, "Performance Evaluation of Sparse Banded Filter Matrices using content based image retrieval", Institute of Integrative Omics and Applied Biotechnology, Vol.7, Issue 3, PP.11-18, 2016.

[10] M. S. Navabi and Z. A. Brooghani, "ContentBased Image Retrieval through Combined Data of Color Moment and Texture", IJCSNS International Journal of Computer Science and Network Security, Vol.17 No.2, February 2017.

[11] M. Deswal and N. Sharma,"A Fast HSV Image Color and Texture Detection", International Journal of Science and Research, 2012. 
[12] M. Soni and P. Singh,"Content Based Image Retrieval Using Combined Gabor and Image Features", International Journal of Computer Science and Technology, Vol.7, Issue 3, July Sept 2016.

[13] A. Singla1and M. Garg,"CBIR Approach Based On Combined HSV, Auto Correlogram, Color Moments and Gabor Wavelet", International Journal Of Engineering And Computer Science, Vol.3, Issue 10 October, PP. 9007-9012, 2014.

[14] H. B. Jehlol, "Image Retrieval Using Association Rules", M.Sc. thesis, Iraqi Commission for Computers And Informatics, Informatics Institute for Postgraduate Studies , Iraq, 2013.

[15] V. Duraisamy and R. B. Durai, "Sequential Minimal Optimization: A Fast Algorithm for Training Support Vector Machines", International Journal of Information Sciences and Application,Vol.3, No.2 ,PP. 93-97, 2011.

[16] K. Jayech and M. A. Mahjoub, "Clustering and Bayesian network for image of faces classification", International Journal of Advanced Computer Science and Applications, Special Issue on Image Processing and Analysis, 2011.

[17] K. Goeschel, "Reducing false positives in intrusion detection systems using data-mining techniques utilizing support vector machines, decision trees, and naive Bayes for off-line analysis", Southeast Con., Norfolk, VA, PP. 16, 2016.
[18] J. Han, Micheline and J. Pei, "Data Mining: Concepts and Techniques", Morgan Kaufmann Publishers Inc, USA, 3rd edition, 2012.

[19] S. Senthil, B.G. Deepa and B. Ashwarya , Comparison of Classification Algorithms for Predicting Breast Cancer", IJSRD International Journal for Scientific Research \& Development, REVA University, Bangalore, India, Vol. 4, Issue 12, 2017.

[20] R. A. OMONDI and C. J. RAJAPAKSE, "FPGA Implementations of Neural Networks", 1St edition, Springer publishing company, 2010.

[21] K. Sharma, A. Kaur and S. Gujral, "Brain Tumor Detection Based On Machine Learning Algorithms", International Journal of Computer Applications (0975 - 8887) Vol. 103 - No.1, October, 2014.

[22] M. A. Hall, "Correlation-based Feature Selection for Machine Learning", the university of Waikato, Hamilton, NewZealand, 1999.

[23] A. J. Mouhamd, "Image Compression Using Wavelet Transform", M.Sc. thesis, College of Science, The University of Al-Mustansiriyah, 2005. 


\title{
خوارزميات تصنيف كفوءة لأسترجاع الصور اعتماداً على الخصائص اللونية والنسيجية
}

\author{
ايمان عبد الجبار سعد

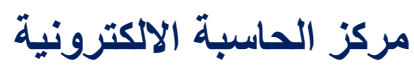 \\ الجامعة المستنصرية، بغداد، العراق الاكتئة
}

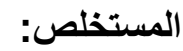

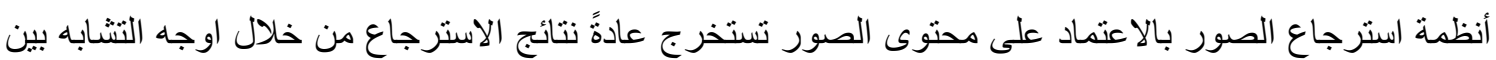

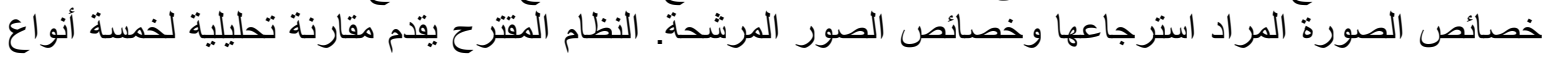

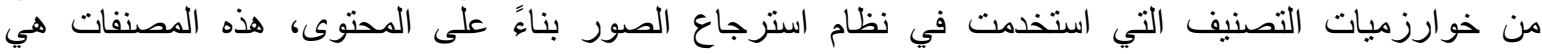
Multilayer Perceptron (MP), Sequential Minimal Optimization (SMO), Random Forest (RF), Bayes Network (BN) and Iterative Classifier Optimizer (ICO)

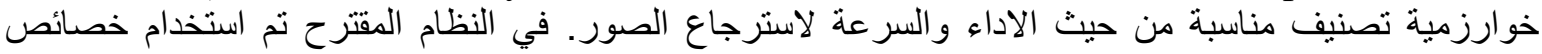

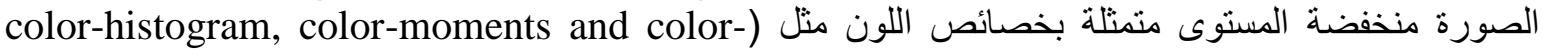

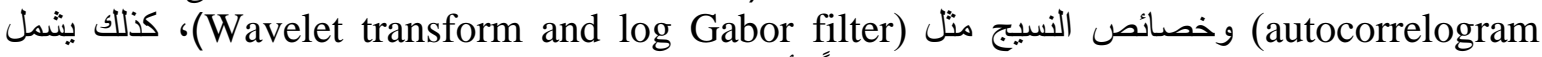

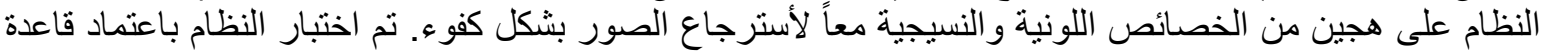

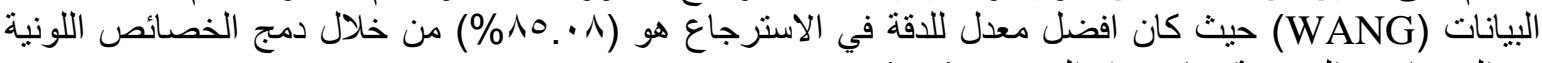

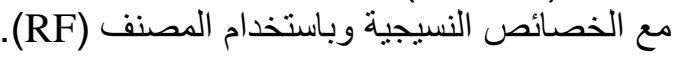

